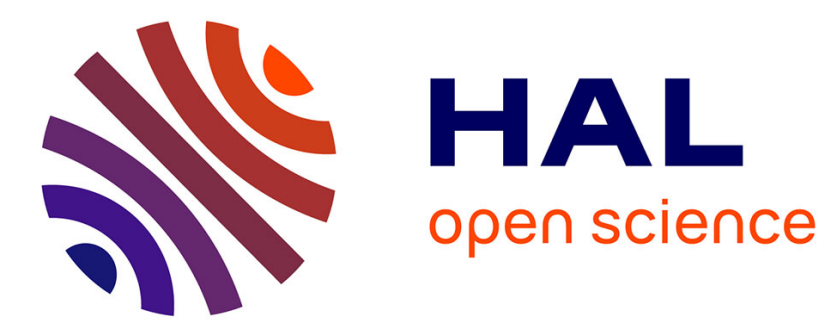

\title{
Présentation et validation du programme OSIRIS de simulation bidimensionnelle des processus technologiques
}

N. Guillemot, P. Chenevier, P. Deroux-Dauphin, J.P. Gonchond

\section{To cite this version:}

N. Guillemot, P. Chenevier, P. Deroux-Dauphin, J.P. Gonchond. Présentation et validation du programme OSIRIS de simulation bidimensionnelle des processus technologiques. Revue de Physique Appliquée, 1984, 19 (12), pp.987-995. 10.1051/rphysap:019840019012098700 . jpa-00245295

\section{HAL Id: jpa-00245295 https://hal.science/jpa-00245295}

Submitted on 1 Jan 1984

HAL is a multi-disciplinary open access archive for the deposit and dissemination of scientific research documents, whether they are published or not. The documents may come from teaching and research institutions in France or abroad, or from public or private research centers.
L'archive ouverte pluridisciplinaire HAL, est destinée au dépôt et à la diffusion de documents scientifiques de niveau recherche, publiés ou non, émanant des établissements d'enseignement et de recherche français ou étrangers, des laboratoires publics ou privés. 
Classification

Physics Abstracts

$05.60-61.70-02.60$

\title{
Présentation et validation du programme OSIRIS de simulation bidimensionnelle des processus technologiques
}

\author{
N. Guillemot, P. Chenevier, P. Deroux-Dauphin (*) et J. P. Gonchond (**) \\ Laboratoire de Physique des Composants à semiconducteurs $\left({ }^{+}\right)$, ENSERG, \\ 23, rue des Martyrs, 38031 Grenoble Cedex, France
}

$\left(^{*}\right)$ THOMSON-EFCIS, rue des Martyrs, BP 127, 38019 Grenoble Cedex, France

$(* *)$ CNET-CNS, Chemin du Vieux-Chêne, BP 398, 38243 Meylan, France

(Reçu le 16 mai 1984, révisé le 30 août, accepté le 12 septembre 1984)

\begin{abstract}
Résumé. - Nous présentons le programme bidimensionnel de simulation des processus technologiques, OSIRIS. Les modèles utilisés pour l'implantation ionique, la croissance d'oxyde et la redistribution des dopants sont d'abord développés. Nous décrivons d'autre part la méthode de résolution de l'équation de diffusion par différences finies. Pour terminer, une comparaison est faite entre des profils de bore obtenus par simulation et des profils expérimentaux à deux dimensions mesurés par EBIC.
\end{abstract}

\begin{abstract}
In the present paper, the two-dimensional process simulator OSIRIS is described. The models used for ion-implantation, oxide growth and redistribution of dopants are developed. Then the numerical method of finite differences used to solve the diffusion equation is discussed. Finally a comparison between boron simulated profiles and two-dimensional experimental data obtained by EBIC measurements is given.
\end{abstract}

\section{Introduction.}

Depuis plusieurs années, les technologues des circuits intégrés au silicium ont éprouvé le besoin de disposer de programmes de simulation des processus de fabrication. Dans une première étape, un certain nombre de programmes unidimensionnels $[1,2]$ ont été développés. Malgré leurs imperfections, ces programmes sont universellement utilisés. Cependant, la réduction des dimensions des composants des circuits intégrés et l'augmentation de leur densité rendent insuffisants les modèles unidimensionnels. Citons, à titre d'exemple, la non-uniformité de l'épaisseur d'oxyde au voisinage des masques de nitrure (formation d'un « bec d'oiseau ») et la diffusion latérale, qui sont typiquement de nature bidimensionnelle et jouent un rôle important dans le fonctionnement des dispositifs.

Un certain nombre de programmes de simulation à deux dimensions commencent à voir le jour [3-7]. Cependant, la plupart nécessitent des ordinateurs de grande capacité et des temps de calcul importants, surtout quand ils utilisent une méthode d'éléments finis. D'autres, au contraire, mettent en œuvre des méthodes qui ne peuvent pas simuler correctement la

$\left(^{+}\right)$ERA CNRS 659. redistribution des dopants dans le cas de fortes concentrations [3]. De plus, l'absence presque totale de mesures directes de profils expérimentaux bidimensionnels fait que ces programmes ne sont pas validés finement en ce qui concerne la diffusion latérale.

Nous présentons ici un programme de simulation bidimensionnelle, OSIRIS (Outil pour la SImulation de la Redistribution des Impuretés dans le Silicium), de taille réduite, d'utilisation facile, qui prend en compte actuellement les principales étapes de fabrication des circuits intégrés au silicium dans le cas d'une impureté, l'extension à deux impuretés étant en cours [8]. Nous présentons également sa validation expérimentale à deux dimensions par le relevé de profondeurs de jonction obtenues après redistribution en utilisant des mesures EBIC.

Dans une première partie, nous présentons les modèles physiques utilisés pour la simulation des principales étapes technologiques de fabrication des circuits intégrés : dopage du silicium par implantation ionique ou par prédépôt, redistribution des impuretés sous atmosphère inerte ou oxydante, avec formation éventuelle d'un «bec d'oiseau ». Nous abordons ensuite l'aspect numérique et informatique : traitement de la frontière mobile à l'interface $\mathrm{SiO}_{2} / \mathrm{Si}$, discrétisation de l'équation de diffusion et résolution du système obtenu. Dans une dernière 
partie nous présentons la validation bidimensionnelle de ce programme grâce à des mesures expérimentales de profondeurs de jonction obtenues dans le cas d'une structure LOCOS [9].

\section{Description et modélisation physique des processus de fabrication.}

Le dopage de la tranche de silicium par implantation ionique ou par prédépôt d'impuretés, et son recuit sous atmosphère inerte ou oxydante constituent les principales étapes de fabrication des circuits intégrés silicium [10]. Le programme OSIRIS simule ces différentes étapes et leur enchaînement, afin d'obtenir le profil des dopants au cours d'un processus de fabrication. Nous décrivons par la suite la physique et la modélisation de ces étapes.

2.1 LE DOPAGE DU SILICIUM. - Dans les processus de fabrication des circuits intégrés, la tranche de silicium est dopée par implantation ionique ou par prédépôt d'impuretés. Quatre impuretés intéressent principalement le silicium : le bore, le phosphore, l'antimoine et l'arsenic. Le prédépôt étant un cas particulier de redistribution d'impuretés, nous ne développerons ici que les modèles utilisés pour simuler leur implantation ionique.

La géométrie du dispositif sur lequel on modélise l'implantation ionique est présenté figure 1 : sur la tranche de silicium un masque, opaque ou non, a été déposé.

La densité de probabilité qu'un ion, percutant la surface en $y$, se loge en $\left(x_{0}, y_{0}\right)$ est $P_{X}\left(x_{0}\right) P_{Y}\left(y_{0}-y\right)$ où $P_{X}$ est la densité de probabilité suivant $x$ (verticale) et $P_{Y}$ la densité de probabilité suivant $y$ (latérale). Ceci en admettant que les probabilités suivant $x$ et suivant $y$ sont indépendantes.

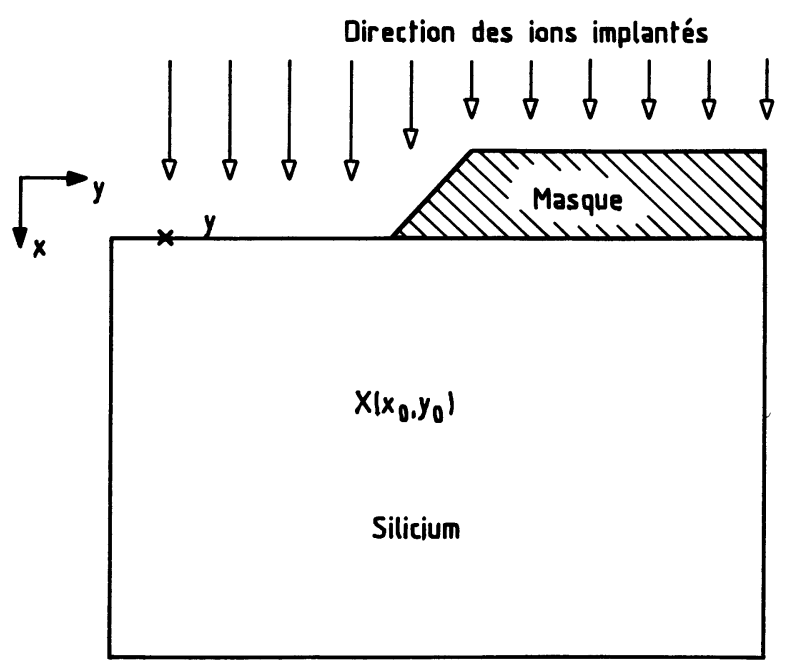

Fig. 1. - Géométrie de la tranche de silicium sur laquelle on modélise l'implantation ionique.

[Silicon wafer geometry for modeling ion-implantation.]
D'après Furukawa [11], on fait l'hypothèse d'une distribution latérale Gaussienne, d'écart-type $\Delta R_{p y}$. $P_{Y}\left(y-y_{0}\right)$ se met alors sous la forme :

$$
P_{Y}\left(y-y_{0}\right)=\frac{1}{\sqrt{2 \pi} \Delta R_{p y}} \exp -\left[\frac{y-y_{0}}{\sqrt{2} \Delta R_{p y}}\right]^{2} \text {. }
$$

D'autre part, des mesures expérimentales permettent d'observer la distribution verticale des ions implantés. On s'aperçoit alors que la distribution gaussienne donnée par les théoriciens donne des résultats peu satisfaisants. La densité de probabilité suivant $x$, soit $P_{x}$, est donc obtenue empiriquement : le profil de bore est bien simulé par une Peason IV modifiée d'une queue exponentielle $[1,12,13]$, celui de l'arsenic, du phosphore et de l'antimoine par une bi-gaussienne [1, 17].

Lorsqu'un masque a été déposé sur la tranche de silicium, le modèle de Runge [15] permet de prendre en compte la pénétration éventuelle des impuretés à travers le masque grâce au modèle suivant : quand le masque n'est pas opaque, on peut le remplacer par une surépaisseur de silicium $d(y)$. Si $d_{\mathrm{r}}(y)$ est l'épaisseur réelle du masque au point $y, R_{\mathrm{pm}}$ la profondeur moyenne de pénétration des ions dans le masque et $R_{\mathrm{ps}}$ la profondeur moyenne de pénétration des ions dans le silicium, cette surépaisseur $d(y)$ est égale à :

$$
d(y)=d_{\mathrm{r}}(y) \frac{R_{\mathrm{ps}}}{R_{\mathrm{pm}}} .
$$

La concentration $N\left(x_{0}, y_{0}\right)$ en chaque point $\left(x_{0}, y_{0}\right)$ du silicium est alors donnée par :

$$
\begin{aligned}
N\left(x_{0}, y_{0}\right)=\frac{N_{\mathrm{d}}}{\sqrt{2 \pi} \Delta R_{p y}} \int_{R} \exp & -\left[\frac{y-y_{0}}{\sqrt{2} \Delta R_{p y}}\right]^{2} \times \\
& \times P_{X}\left(x_{0}+d(y)\right) \mathrm{d} y
\end{aligned}
$$

où $N_{\mathrm{d}}$ est la dose d'impuretés implantées par unité de surface.

$\mathrm{Du}$ point de vue numérique, on divise le masque en fines tranches $\left[y_{k}, y_{k+1}\right]$, d'épaisseur moyenne $d_{k}$, éventuellement nulle (Fig. 2), et l'on calcule en chaque point $\left(x_{0}, y_{0}\right)$ du silicium la concentration $N_{k}\left(x_{0}, y_{0}\right)$ en impuretés, due à l'implantation à travers cette tranche de masque. On somme ensuite sur toutes les tranches de masque possibles.

Dans le cas d'un masque opaque, positionné en $L$ (Fig. 3), l'expression précédente se simplifie en :

$$
N\left(x_{0}, y_{0}\right)=\frac{N_{\mathrm{d}}}{2 \sqrt{2 \pi} \Delta R_{p y}} P_{X}\left(x_{0}\right) \operatorname{erfc}\left[\frac{L-y_{0}}{\sqrt{2} \Delta R_{p y}}\right] .
$$

Les figures 4 et 5 montrent les profils de bore et d'arsenic obtenus par simulation dans le cas d'un masque rendu opaque par dépôt d'une résine. 


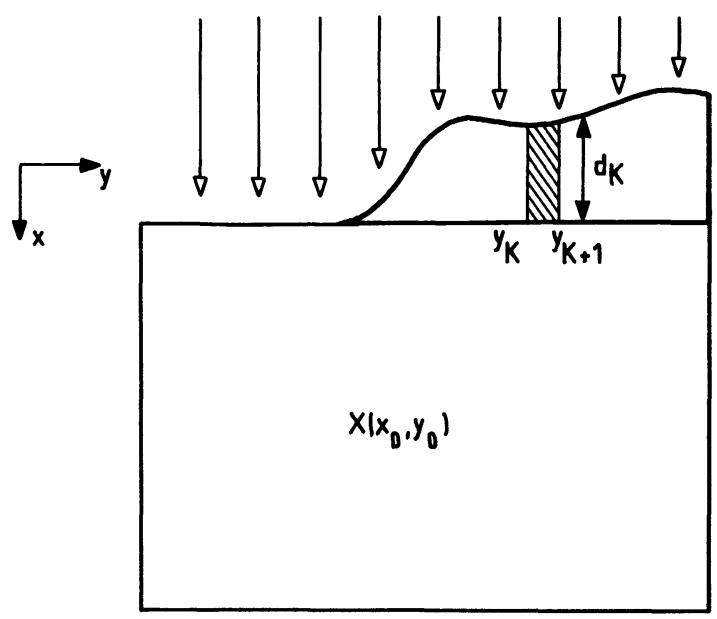

Fig. 2. - Géométrie de la tranche de silicium dans le cas d'un masque non opaque.

[Geometry of the silicon wafer with an arbitrary shaped mask edge.]

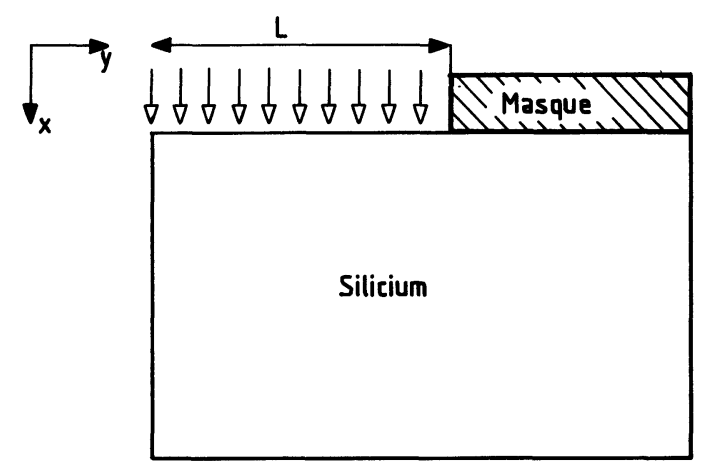

Fig. 3. - Géométrie de la tranche de silicium avec un masque opaque.

[Geometry of the silicon wafer with a vertical shaped mask edge.]

2.2 LE RECUIT. - Le recuit de la tranche de silicium en atmosphère inerte ou oxydante redistribue les impuretés dans le semiconducteur. On considère un type d'impureté $(k)$ diffusant dans un processus multiimpuretés. L'équation qui régit son transport est donnée par l'équation de diffusion, issue des deux lois de Fick [15]. Elle s'écrit, en chaque point $(x, y)$ du semiconducteur, en tenant compte du champ électrique :

$$
\begin{aligned}
& \frac{\delta N_{k}}{\delta t}(x, y)= \\
& =\left\{\operatorname { d i v } \left(D _ { k } \left(\operatorname{grad} N_{k}+Z_{k} \frac{\frac{N_{k}}{2 n_{i}}}{\left.\left.\left.\sqrt{\frac{M^{2}}{4 n_{i}^{2}}+1} \operatorname{grad} M\right)\right)\right\}(x, y)}\right.\right.\right.
\end{aligned}
$$

où $N_{k}$ est la concentration de l'impureté $k$.

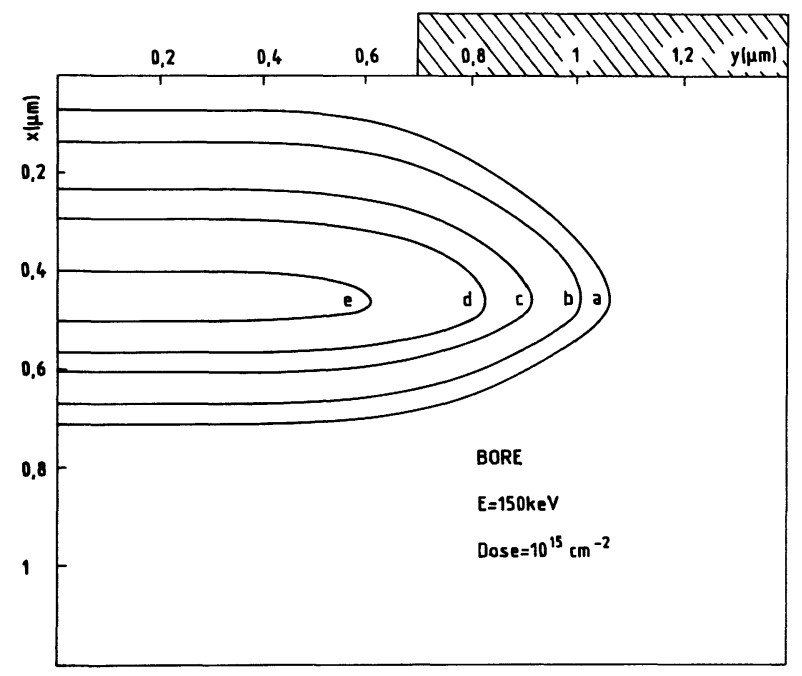

Fig. 4. - Contours d'isoconcentration de bore après implantation ionique (Pearson IV modifiée selon $x$, Gaussienne selon $y$ ). Energie : $150 \mathrm{keV}$, Dose : $1 \times 10^{15}$ atomes/ $\mathrm{cm}^{2}$. (a) $N=4 \times 10^{17}$ atomes $/ \mathrm{cm}^{3}$. (b) $N=1 \times 10^{18}$ atomes/ $\mathrm{cm}^{3}$. (c) $N=4 \times 10^{18}$ atomes $/ \mathrm{cm}^{3}$. (d) $N=1 \times 10^{19}$ atomes/ $\mathrm{cm}^{3}$. (e) $N=4 \times 10^{19}$ atomes $/ \mathrm{cm}^{3}$.

[Equi-density contours of boron after ion-implantation (Modified Pearson IV in the $x$-direction, Gaussian function in the $y$-direction).]

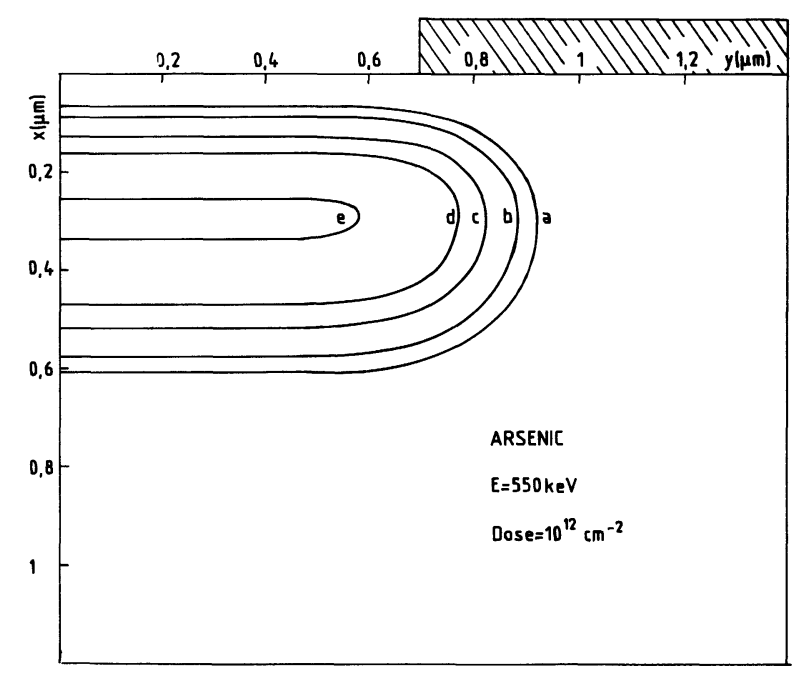

Fig. 5. - Contours d'isoconcentration d'arsenic après implantation ionique (Bi-Gaussienne selon $x$, Gaussienne selon y). Energie : $550 \mathrm{keV}$, Dose : $1 \times 10^{12}$ atomes $/ \mathrm{cm}^{2}$. (a) $N=4 \times 10^{14}$ atomes $/ \mathrm{cm}^{3}$. (b) $N=1 \times 10^{15}$ atomes $/ \mathrm{cm}^{3}$. (c) $N=4 \times 10^{15}$ atomes $/ \mathrm{cm}^{3}$. (d) $N=1 \times 10^{16}$ atomes $/ \mathrm{cm}^{3}$. (e) $N=4 \times 10^{16}$ atomes $/ \mathrm{cm}^{3}$.

[Equi-density contours of arsenic after ion-implantation (Joined half-Gaussian distribution in $x$-direction, Gaussian in $y$-direction).] reté $k$.

$D_{k}$ est le coefficient de diffusion effectif de l'impu-

$Z_{k}$ est l'état de charge $(+1$ pour les accepteurs, -1 pour les donneurs). 
$n_{i}$ est la concentration intrinsèque.

$M=\sum_{k} Z_{k} N_{k}$ (concentration nette en accepteurs).

On a fait l'hypothèse de neutralité et d'ionisation complète des impuretés.

Pour les quatre impuretés, on se place dans le cas d'un mécanisme de diffusion purement lacunaire. Le coefficient de diffusion effectif peut alors se mettre sous la forme [16] :

$$
D_{k}=D_{k}^{0} \frac{1+\beta_{k} f_{k}}{1+\beta_{k}}
$$

où $D_{k}^{0}$ est le coefficient de diffusion intrinsèque de l'impureté $k$.

$\beta_{k}$ est un coefficient phénoménologique (on prend en général $\beta_{k}=25$ pour les accepteurs [17] et $\beta_{k}=100$ pour les donneurs).

$f_{k}=p / n_{i}$ ou $n / n_{i}$ suivant que l'impureté est acceptrice ou donatrice respectivement.

Le profil des impuretés après redistribution est obtenu par résolution de cette équation de diffusion, compte tenu des conditions aux limites.

2.3 LA CROISSANCE D'OXYDE. - Lors d'un recuit de la tranche de silicium en atmosphère oxydante, la silice croît et prend sous le masque une forme très particulière, dite en « bec d'oiseau » (Fig. 6) [18]. La résolution numérique des équations qui régissent la formation d'oxyde donne de bons résultats mais s'avère longue et difficile $[19,20]$. Il est préférable d'utiliser, dans la mesure du possible, un bon modèle empirique : dans le cas classique d'une structure LOCOS (Fig. 7), Dutton et al. ont donné un modèle analytique satisfaisant pour certaines épaisseurs de nitrure et de piédestal [21]. Nous l'avons utilisé dans le programme OSIRIS. Notant $Z_{1}(y, t)$ l'équation du bord inférieur de la silice et $Z_{2}(y, t)$ l'équation du bord supérieur (Fig. 7), le modèle donne l'expression de $Z_{1}$ et $Z_{2}$ en fonction de l'épaisseur $T_{0}$ de piédestal, de l'épaisseur $T_{n}$ de nitrure et de l'épaisseur $Z_{0}$ d'oxyde donné par le modèle de Deal et Grove [22]. Il suppose une température et une pression constante lorsque l'oxyde se forme. Dans le cas où l'un de ces deux paramètres est variable on peut calculer pas à pas, par différentiation, l'évolution de $Z_{1}$ et $Z_{2}$ au cours du temps.

3. Calcul numérique du profil des impuretés diffusantes.

Dans cette partie, nous abordons le problème de la résolution de l'équation de diffusion sur un domaine qui est variable si l'on se place dans le cas d'une redistribution en atmosphère oxydante. Les géométries initiales de la tranche de silicium sur lesquelles les différents processus peuvent être simulés sont du type illustré figure 8 . On suppose que le domaine est symétrique, ou qu'il est suffisamment étendu en $y$ pour que l'on puisse appliquer les conditions de symétrie en $y=0$ et $y=Y_{m}$.

3.1 LE TRAITEMENT DE LA FRONTIÈRE MOBILE. Lorsque l'on se place dans le cas d'un recuit de la tranche de silicium en atmosphère oxydante, la croissance d'oxyde rend mobile la frontière qui correspond à l'interface silice/silicium (Fig. 7). Pour

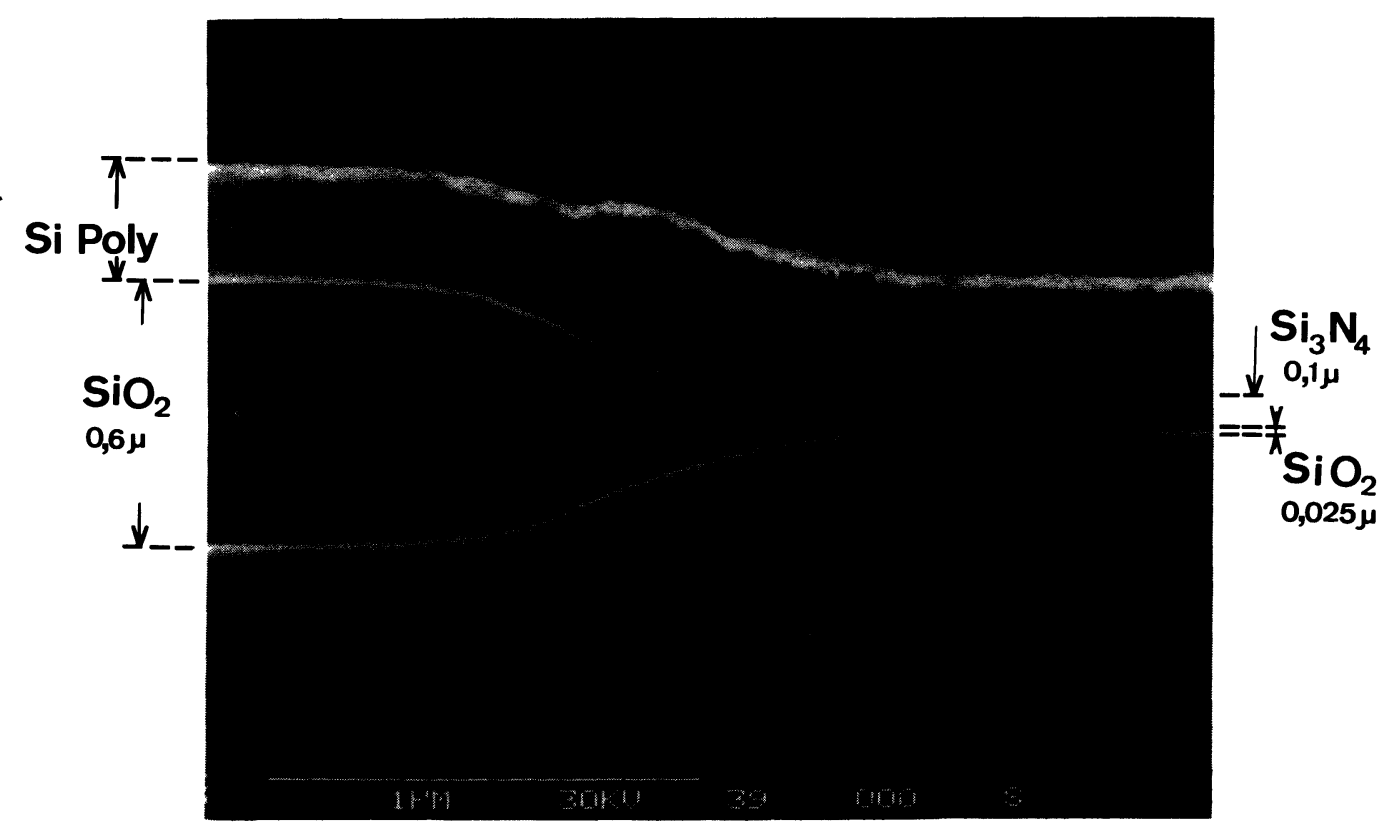

Fig. 6. - Croissance d'oxyde en forme de « bec d'oiseau » dans le cas d'une structure LOCOS.

[Formation of the bird's beak in the local oxidation process (LOCOS).] 


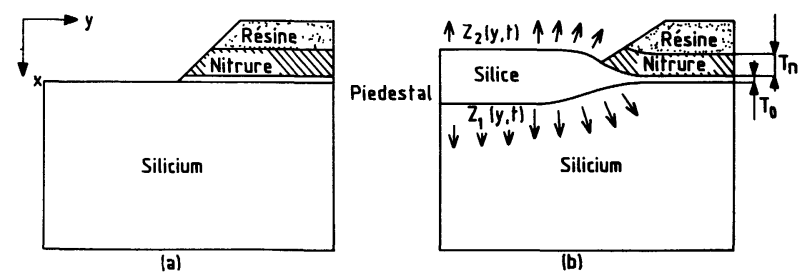

Fig. 7. - Evolution de la frontière silice/silicium dans le cas d'une structure LOCOS : (a) Géométrie initiale de la tranche de silicium après oxydation. (b) Géométrie de la tranche de silicium après oxydation. $Z_{1}(y, t)=$ équation du bord inférieur de la silice. $Z_{2}(y, t)=$ équation du bord supérieur de la silice.

[Evolution of the $\mathrm{SiO}_{2} / \mathrm{Si}$ boundary in the local oxidation process : (a) Initial silicon wafer geometry. (b) Geometry after oxidation. $Z_{1}(y, t)=$ oxide contour at the silicon/oxide interface. $Z_{2}(y, t)=$ oxide contour at the ambient/oxide interface.]

l'instant, le programme OSIRIS traite le cas de la redistribution d'une seule impureté. Son équation de diffusion s'écrit alors :

$$
\frac{\delta N}{\delta t}(x, y, t)=\operatorname{div}(D(N) \operatorname{grad} N)(x, y, t)
$$

sur le domaine $\Omega$ illustré figure 9 .

$N(x, y, t)$ est la concentration de l'impureté au point $(x, y)$ à l'instant $t$, et $D$ le coefficient de diffusion qui tient compte du champ électrique.

On note $Y_{m}$ la largeur de la tranche de silicium, $L_{0}$ son épaisseur utile, $X^{0}(y, t)$ l'équation de la frontière supérieure de la tranche (interface silice/ silicium). $\Omega$ est alors décrit par :

$$
\left[\begin{array}{l}
X^{0}(y, t)<x<L_{0} \\
0<y<Y_{m}
\end{array} .\right.
$$

Les conditions aux limites sont données :

- En appliquant les conditions de symétries :

$$
\left.\begin{array}{l}
\left.\frac{\delta N}{\delta y}\right|_{y=0}=0 \\
\left.\frac{\delta N}{\delta y}\right|_{y=Y}=0
\end{array}\right] X^{0}(y, t)<x<L_{0} .
$$

- En supposant le domaine choisi suffisamment profond :

$$
\left.\frac{\delta N}{\delta x}\right|_{x=L_{0}}=0 \quad 0<y<Y_{m} .
$$

Pour écrire la condition à l'interface silice/silicium [23], on néglige la diffusion dans l'oxyde, et l'on suppose qu'à tout instant la ségrégation entre les deux milieux est celle de l'équilibre, ce qui donne :

$$
\left.\frac{\delta N}{\delta x}\right|_{x=X^{0}(y, t)}=(k-m) V \cdot n N
$$

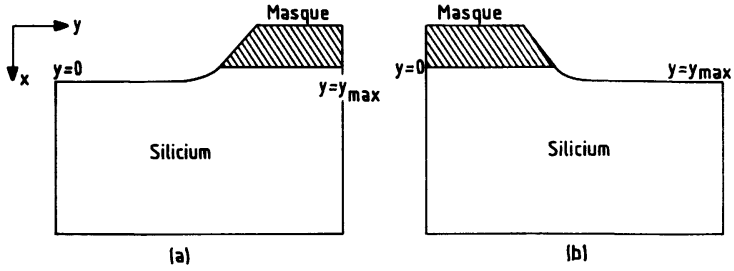

Fig. 8. - Géométries de la tranche de silicium sur laquelle on simule les processus : (a) Masque à droite. (b) Masque à gauche.

[Geometries used for process, modelling : (a) Oxidation mask on the right side. (b) Oxidation mask on the left side.]

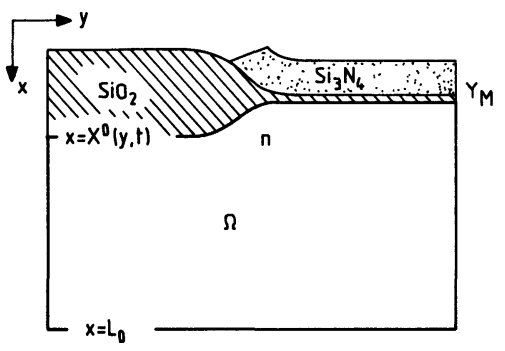

Fig. 9. - Domaine $\Omega$ sur lequel l'équation de diffusion est résolue.

[Physical domain $\Omega$ used to solve the diffusion equation.]

où $k$ est le coefficient de ségrégation à l'interface silice/silicium,

$V$ est la vitesse de croissance de l'oxyde,

$n$ est la normale à la frontière,

$m$ est le rapport de l'épaisseur de silicium consommé sur l'épaisseur d'oxyde formé.

Pour résoudre l'équation de diffusion sur un domaine $\Delta$ rectangulaire et fixe au cours du temps, il suffit de faire le changement de variable $x$ en $\xi$ [23] avec :

$$
\xi=\frac{x-X_{0}^{0}}{L_{0}-X^{0}(y, t)} L_{0}
$$

On pose : $L=L_{0}-X^{0}(y, t)$, l'équation de diffusion s'écrit, dans le nouveau système de coordonnées $(\xi, y, t)$ :

$$
\begin{aligned}
& \frac{\delta N}{\delta t}(\xi, y, t)= \\
& =\left\{A A \frac{\delta}{\delta \xi}\left(D \frac{\delta N}{\delta \xi}\right)+B B \frac{\delta}{\delta y}\left(D \frac{\delta N}{\delta y}\right)+\right. \\
& \left.+A B\left(\frac{\delta}{\delta \xi}\left(D \frac{\delta N}{\delta y}\right)+\frac{\delta}{\delta y}\left(D \frac{\delta N}{\delta \xi}\right)\right)+A \frac{\delta N}{\delta \xi}\right\}(\xi, y, t)
\end{aligned}
$$




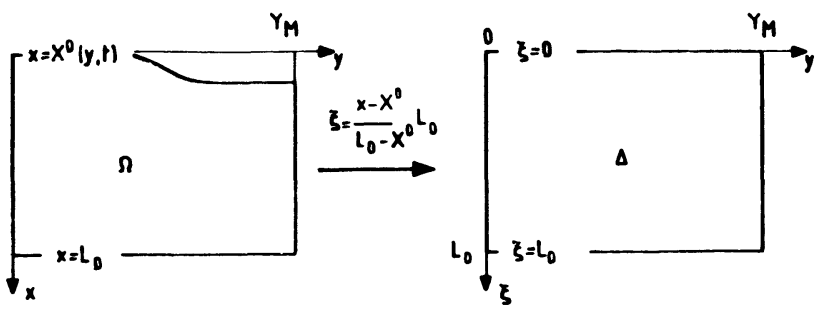

Fig. 10. - Transformation du domaine $\Omega$ variable au cours du temps en un domaine $\Delta$ rectangulaire fixe, à l'aide du changement de variable $x$ en $\xi$.

[Transformation of the physical domain $\Omega$ into a fixed time invariant rectangular domain $\Delta$ by means of a coordinate transformation $x$ into $\xi$.]

$$
\begin{aligned}
A A & =\frac{L_{0}^{2}+\left(\left(L_{0}-\xi\right) X_{y}^{0}\right)^{2}}{L^{2}} \\
B B & =1 \\
A B & =-\left(L_{0}-\xi\right) \frac{X_{y}^{0}}{L} \\
A & =\frac{L_{0}-\xi}{L^{2}}\left(L X_{t}^{0}-D\left(L X_{y y}^{0}+2\left(X_{y}^{0}\right)^{2}\right)\right) .
\end{aligned}
$$

Avec les notations habituelles :

$$
\begin{aligned}
X_{y}^{0} & =\frac{\delta X^{0}}{\delta y} \\
X_{y y}^{0} & =\frac{\delta^{2} X^{0}}{\delta y^{2}} \\
X_{t}^{0} & =\frac{\delta X^{0}}{\delta t} .
\end{aligned}
$$

Les conditions limites sont données par :

$$
\begin{gathered}
\left.\frac{\delta N}{\delta y}\right|_{y=0}=0 \quad 0<\xi<L_{0} \\
\left.\quad \frac{\delta N}{\delta y}\right|_{y=Y_{m}}=0 \quad 0<\xi<L_{0} \\
\left.\quad \frac{\delta N}{\delta \xi}\right|_{\xi=L_{0}}=0 \quad 0<y<Y_{m} \\
D \frac{L_{0}}{L}\left(1+\left(X_{y}^{0}\right)^{2}\right) \frac{\delta N}{\delta \xi}-D X_{y}^{0} \frac{\delta N}{\delta y}=\left.(k-m) V N\right|_{\xi=0} \\
\quad 0<y<Y_{m} .
\end{gathered}
$$

Pour cette dernière condition, on a supposé que la vitesse de croissance de l'oxyde n'avait qu'une composante $V$ suivant $x$.

3. 2 LA RÉSOLUTION NUMÉRIQUE. - Pour résoudre l'équation de diffusion, le domaine $\Delta$ est d'abord discrétisé par une grille rectangulaire de $N_{\xi} \times N_{y}$ points, le pas de cette grille peut être affiné dans les régions où les gradients de concentration sont importants (Fig. 11).

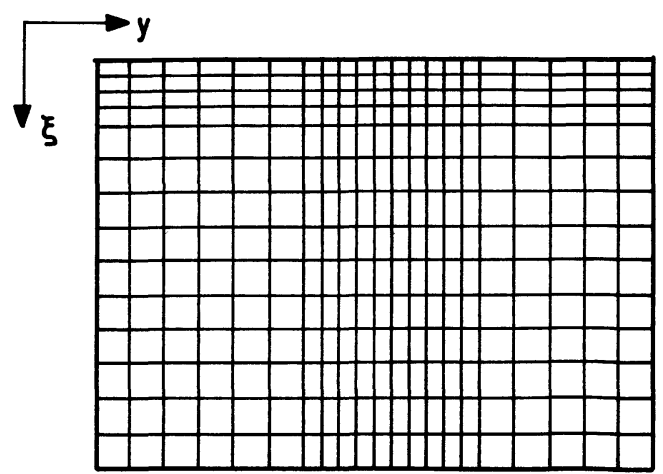

(a)

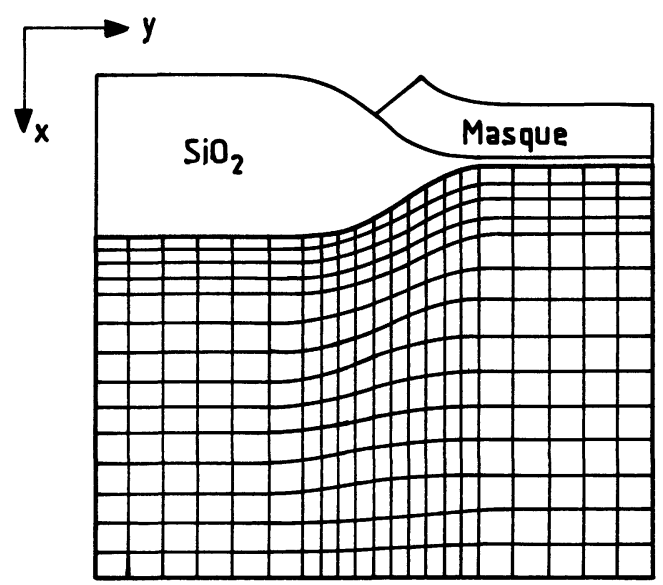

(b)

Fig. 11. - Discrétisation du domaine pour la résolution de l'équation de diffusion par différences finies : (a) Grille sur le domaine transformé $\Delta$. (b) Grille sur le domaine initial $\Omega$, avec resserrement du pas à l'interface silice/silicium, et aux alentours du bord du masque.

[Discretization of the computational domain used to solve the diffusion equation by finite differences. (a) Grid on the transformed domain $\Delta$. (b) Grid on the initial domain $\Omega$ showing a smaller partition in the vicinity of the oxide/silicon interface and of the mask edge.]

On cherche alors la concentration $N_{i, j}$ en chaque noeud $(i, j)$ du maillage. La méthode classique des différences finies [24] est utilisée pour discrétiser le second membre de l'équation (dérivées spatiales), la dérivée temporelle ( $1^{\mathrm{er}}$ membre de l'équation) est approchée par un schéma de Cranck-Nicholson [25].

$\mathrm{Au} n+1$-ième pas temporel, on cherche les concentrations aux nœuds du maillage, connaissant les concentrations au $n$-ième pas. On est alors amené à résoudre un système de $N_{\xi} \times N_{y}$ équations à $N_{\xi} \times N_{y}$ inconnues, soit :

$$
A^{n+1} N^{n+1}=B^{n}
$$

où $N^{n+1}$ est le vecteur des concentrations en chaque point à l'instant $n+1$. Le système est non linéaire : les coefficients de la matrice $A$ dépendent des concentrations au temps $n+1$. La résolution se fait par une 
méthode de Gauss-Seidel (du fait de la forte nonlinéarité du problème, on ne pouvait employer une méthode de surrelaxation avec un paramètre d'accélération), en modifiant les coefficients au fur et à mesure des itérations.

$\mathrm{Du}$ point de vue informatique, le choix de ces méthodes nous a permis de faire un programme de taille réduite avec des temps de calcul très raisonnables.

\section{Validation du programme.}

4.1 MESURES EXPÉRIMENTALES EFFECTUÉES SUR DISPositifs. - Nous résumons ici les résultats expérimentaux obtenus dans le cas de l'étude portée en référence [9]. Elle caractérise la diffusion du bore selon deux dimensions (latérale et verticale), dans le cas d'une structure LOCOS.

Les techniques de caractérisation utilisées sont les suivantes : la profondeur de jonction est obtenue en fonction de $y$ en mesurant le courant induit par faisceau d'électrons (EBIC) et par révélation chimique; dans les régions de nature quasi unidimensionnelle on fait en plus des mesures par sonde ionique (SIMS). Les mesures sont effectuées pour 4 processus qui comportent les étapes suivantes :

- Une implantation ionique de bore $(E=80 \mathrm{keV}$, dose $=3 \times 10^{12}$ atomes $/ \mathrm{cm}^{2}$ ) avec un masque de nitrure rendu opaque par dépôt d'une résine.

- Un cycle d'oxydation : le bore est d'abord redistribué sous atmosphère inerte, avec une montée en température jusqu'à $920^{\circ} \mathrm{C}$, puis sous atmosphère oxydante humide. Les 4 processus diffèrent par la pression de cette atmosphère oxydante $(1,5,15$, $20 \mathrm{bar})$. Dans le cas des hautes pressions $(5,15$, 20 bar), la pression augmente linéairement à partir de 1 bar jusqu'à la valeur désirée, puis elle reste constante jusqu'à la fin de l'oxydation. Le temps d'oxydation est ajusté en sorte que dans tous les cas, l'épaisseur d'oxyde formée soit la même.

- Une descente en température et en pression.

4.2 La simulation. - Pour simuler les différents processus, nous avons essayé d'être aussi proche que possible des conditions technologiques, en tenant compte notamment des variations de température et de pression lors des recuits.

Le programme OSIRIS dispose de tables de paramètres par défaut, en particulier pour les paramètres d'implantation $[1,26]$ (profondeur moyenne de pénétration des ions, écarts-types, etc.). Or, si pour les fortes doses de bore $\left(>10^{14}\right.$ atomes $\left./ \mathrm{cm}^{2}\right)$, ces paramètres donnent une bonne simulation du profil d'implantation, dans notre cas, la dose étant faible $\left(3 \times 10^{12}\right.$ atomes $\left./ \mathrm{cm}^{2}\right)$, un profil expérimental unidimensionnel par SIMS (Fig. 12) a montré qu'il était nécessaire d'ajuster ces paramètres. Le profil bidimensionnel simulé présente alors un aplatissement prononcé de la forme de la jonction sous le masque (Fig. 13). Les mesures EBIC avaient mis en évidence cette irrégularité, qui fut confirmée par

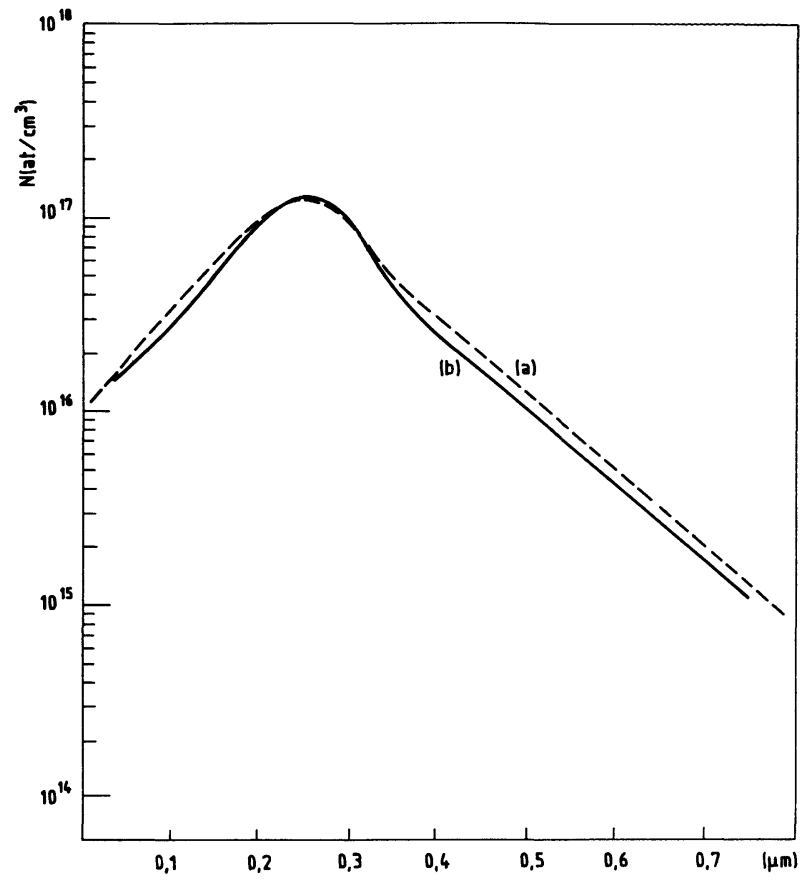

Fig. 12. - Profil unidimensionnel d'implantation de bore : $E=80 \mathrm{keV}$, dose $=3 \times 10^{12}$ atomes $/ \mathrm{cm}^{2}$. (a) Profil expérimental obtenu par SIMS (trait plein). (b) Profil simulé par une Pearson IV modifiée (trait pointillé).

[One-dimensional boron profile after ion-implantation : $80 \mathrm{keV}$, implant $3 \times 10^{12}$ ions per $\mathrm{cm}^{2}$. (a) Concentration profile measured by SIMS (solid line). (b) Concentration profile simulated by a modified Pearson IV (dashed line).]

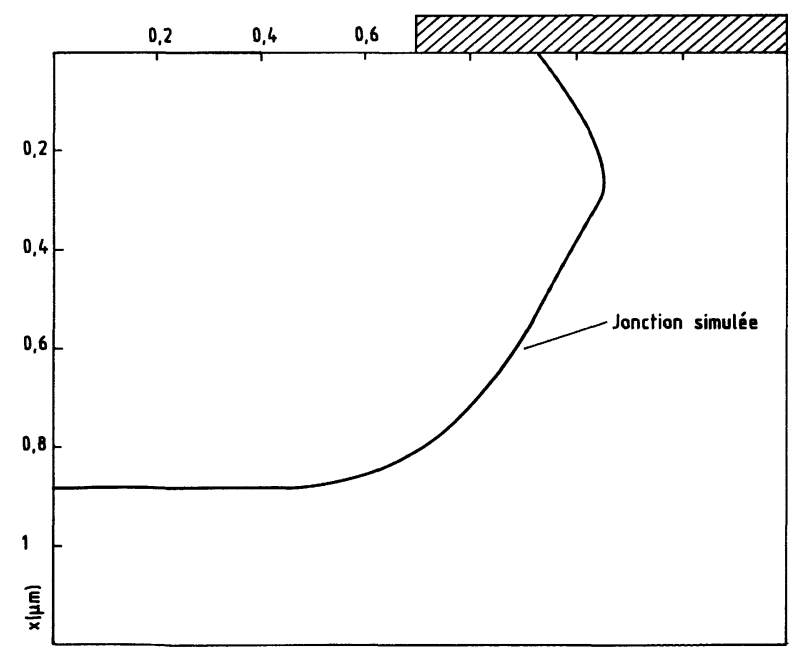

Fig. 13. - Implantation de bore à travers un masque opaque, profondeur de jonction obtenue par simulation (substrat $N: 4 \times 10^{14}$ atomes par $\mathrm{cm}^{3}$ ).

[Simulated junction depth obtained after boron implantation in silicon $\left(N\right.$-substrate $: 4 \times 10^{14}$ ions per $\left.\mathrm{cm}^{3}\right)$.] 
révélation chimique sur les profils expérimentaux à très hautes pressions ( 15 et 20 bar) [9] : les temps de recuit sont alors très courts, le bore ne diffuse pratiquement pas et le profil reste très proche du profil d'implantation. A 1 et 5 bar, les temps de recuit sont longs ( $455 \mathrm{~min}$ et $81 \mathrm{~min}$ respectivement), les profondeurs de jonction obtenues par simulation sont en très bon accord avec les mesures expérimentales (Figs. 14 et 15).

Dans ces simulations, 1500 points ont été utilisés pour discrétiser le domaine. Les temps de calcul sur HB-68, varient alors de $15 \mathrm{~min}$ (processus à 5 bar), à $45 \mathrm{~min}$ (processus à 1 bar).

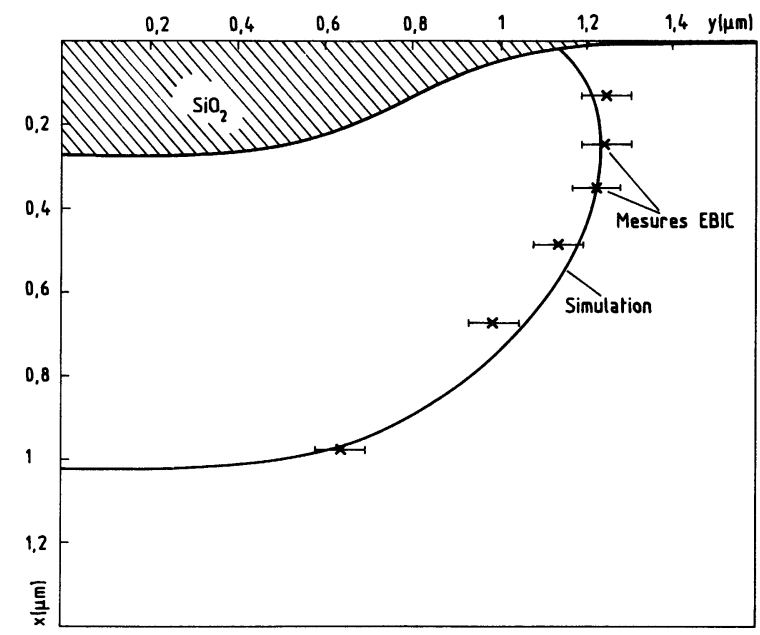

Fig. 14. - Profondeur de jonction obtenue après redistribution du bore en atmosphère oxydante sous pression de 1 bar. Comparaison entre les mesures expérimentales et la simulation.

[Junction depth after boron redistribution in an oxidizing ambient in case of 1 bar pressure. Comparaison between experimental data and simulation profile.]

\section{Conclusion.}

Nous avons présenté dans cet article le programme de simulation à deux dimensions de processus tech- . nologiques OSIRIS. Il simule la plupart des étapes

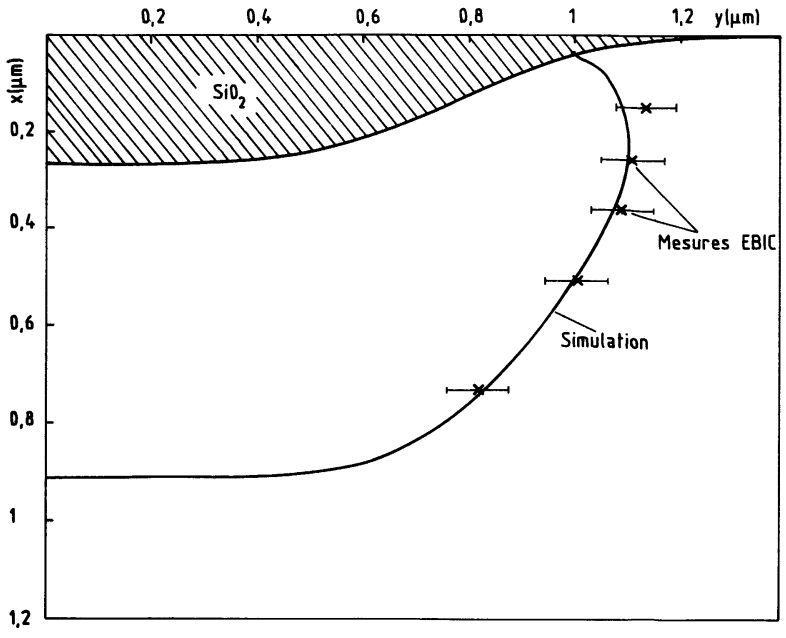

Fig. 15. - Profondeur de jonction obtenue après redistribution du bore sous pression de 5 bar, en atmosphère oxydante. Comparaison entre les mesures expérimentales et la simulation.

[Junction depth after boron redistribution in an oxidizing ambient in case of 5 bar of pressure. Comparaison between experimental data and simulated profile.]

de réalisation des circuits intégrés au silicium, avec une seule impureté actuellement : le passage à plusieurs impuretés est en cours. Les lois d'implantation des impuretés et de croissance d'oxyde sont des lois analytiques obtenues empiriquement. La redistribution des impuretés est du type diffusion lacunaire, avec influence du champ électrique. Tous les paramètres physiques peuvent être pris par défaut. La méthode de résolution des équations de diffusion utilisée (changement de variable pour conserver un domaine rectangulaire fixe, équations aux différences finies résolues par une méthode de GaussSeidel), permet d'avoir un programme de taille réduite avec des temps de calcul raisonnables.

Par ailleurs la mesure à deux dimensions, par méthode EBIC, de la profondeur de jonction du bore implanté avec un masque, puis redistribué sous différentes atmosphères oxydantes, a permis de valider ce programme, en particulier la loi d'implantation et la loi de croissance d'oxyde adoptée. 


\section{Bibliographie}

[1] Antoniadis, D. A., Hansen, S. E., Dutton, R. W. : SUPREM II-A Program for IC Process Modeling and Simulation, TR 5019-2, Stanford Electronics Laboratories, Stanford University, Stanford, CA (June 1978).

[2] Ho, C. P., Plummer, J. D., Hansen, S. E., Dutton, IEEE Trans. ED 30 № 11 (1983) 1438-1452.

[3] Chin, D., Kump, M. R., LeE, H. G.. Dutton, R. W., IEEE Trans. ED 29 № 2 (1982) 336-340.

[4] Maldonado, C. D., Appl. Phys. A31 (1Yjj) 119-138.

[5] Salsburg, K. A., Hansen, H. H., IEEE Trans. ED 29 No 9 (1983).

[6] TieleRT, R., IEEE Trans. ED 27 (1980) 1479-1483.

[7] Collard, D., Decarpigny, J. N., Simulation bidimensionnelle de Processus de dopage, Journées GCIS, Toulouse (Décembre 1983).

[8] Guillemot, N., Chenevier, P., OSIRIS - Presentation and Validation of a New Two-Dimensional Simulator for Technology Process, MIEL 84, NIS, Yugoslavia (May 1984).

[9] Deroux, P., Gonchond, J. P., J. Electrochem. Soc. (à paraître).

[10] Colclaser, R. A., Microelectronics : Processing and Device Design (John Wiley and Sons, New-York) 1980.

[11] Furukawa, S., Matsumura, Ishiwara, I., Japan. J. Appl. Phys. 11 № 2 (1972) 134-142.

[12] Ryssel, H. et al. : IEEE Trans. ED 27 № 8 (1980) 1484-1492.

[13] Mei, L., Dutton, R. W., Hansen, S. E., Modeling on Polycrystalline Silicon Structure for Integrated Fabrication Processes, TR G. 20113, Stanford Electronics Laboratories, Stanford CA (July 1982).
[14] Gibbons, J. F. and Mylroie, S., Appl. Phys. Letters № 11 (1973) 568-569.

[15] Runge, H., Phys. Status Solidi (a) 39 (1977) 595-599.

[16] Nuyts, W. and Van Overstaeten, R., Phys. Status Solidi (a) 15 (1973) 329.

[17] Tsoukalas, D. and Chenevier, P., Spring Meeting of Electrochemical Society, Extended Abstracts 476 (1983).

[18] Shankoff, T. A., Sheng, T. T., Haszko, S. E., MarCUS, R. B., SMITH, T. E., J. Electrochem. Soc. Solid State Science Technol. 127 № 1 (1980) 216-221.

[19] Poncet, A., Two Dimensional Simulation of Local Oxidation in VLSI Processes, VLSI Process and Device Modeling, Vol. 1, Summer Course 83, Heverlee, Belgium (June 1983).

[20] Chin, D., Ho, S. Y., Hu, S. M., Dutron, R. W., HALL, J. L., IEDM 82 (1982) 228-232.

[21] Lee, H. G., Dutton, R. W., IEE Trans. ED 28 № 10 (1981) 744-749.

[22] Deal, B. E. and Grove, A. S., J. Appl. Phys. 36 (1965) 3770-3778.

[23] Maldonado, C. D., J. Appl. Phys. A 31 (1983) 119-138.

[24] FoRsYTHE and WAsow : Finite Difference Methods for Partial Differential Equations (John Wiley and Sons Inc.) 1960.

[25] Cranck, J., Nicholson, P., Proc. Cambridge Philos. Soc. 43 (1947) 50-67.

[26] HACQUeS, G., Mathématiques pour l'informatique 3, Algorithmique Numérique Cours, 121-125 (Librairie Armand Colin, Paris) 1971.

[27] Gibbons, J. F., Johnson, W. S., Mylroie, S. W., Projected Range Statistics (Dowden, Hutchinson and Ross, Stroudsbourh, PA.) 1975. 